\title{
Bisphosphonates: a therapeutic option for knee osteoarthritis?
}

\author{
Willem F Lems ${ }^{1,2}$
}

Osteoarthritis (OA) is one of the rheumatic diseases with the highest incidence and prevalence, which are expected to rise in current ageing populations. ${ }^{1}$ Unfortunately, in contrast to inflammatory rheumatic diseases such as rheumatoid arthritis, ankylosing spondylitis and psoriatic arthritis, there are hardly any treatment options that modify the course of $\mathrm{OA},{ }^{2}$ except for some data on glucosamine and chondrotoine on radiological progression. ${ }^{34}$ In fact, the only instruments we have are symptomatics: analgesics, exercise therapy, weight loss for those with a high body mass index (BMI), intra-articular injections ${ }^{2}$ and orthopaedic surgery for those with severe or end-stage OA.

Nowadays, the disease is regarded as a whole joint disease that affects different structures in and around the joint capsule: the cartilage and also the synovial tissue, subchondral bone, muscles, ligaments and entheses are involved. ${ }^{5}$ In the absence of effective therapeutic options that modify the course of the disease, it is attractive to split up the options for interventions in different targets: cartilage, subchondral bone, synovitis and central pain regulation. ${ }^{5-7}$ Against that background, the study by Neogi $e t a l^{8}$ is very welcome: they showed that the risk of knee replacement (KR) therapy in patients with knee-OA was $25 \%$ lower in bisphosphonate (BP) users than in non-BP users, a remarkable difference, with large potential consequences, at least for elderly individuals with osteoporosis and OA.

\section{DO ANTIRESORPTIVE DRUGS HAVE FAVOURABLE EFFECTS IN OA?}

Recent experimental and clinical studies have demonstrated that changes in subchondral bone and a crosstalk between subchondral bone and cartilage play an

\footnotetext{
1Department of Rheumatology, Amsterdam Rheumatology and Immunology Center, VU University Medical Center, Amsterdam, The Netherlands 2Department of Rheumatology, Reade, Amsterdam, The Netherlands
}

Correspondence to Professor Willem F Lems, Department of Rheumatology, Amsterdam Rheumatology and immunology centre, VU University medical centre, Amsterdam 1007 mb, The Netherlands; wf.lems@vumc.nl important role in the development of $\mathrm{OA},{ }^{9}$ although the pathophysiology and changes in subchondral bone have not been fully elucidated. In the early phase, increased osteoclast activity, elevated bone remodelling and hypomineralisation can be found, ${ }^{79}$ while in later stages low bone turnover and densification of the bone ('bone sclerosis') can be observed. Additionally, bone marrow lesions (BML) can be detected on MRI. ${ }^{59}$

Thus, theoretically, the use of antiosteoporotic drugs that can be regarded as antiresorptive drugs (BPs such as alendronate, risedronate, zoledronic acid, but also denosumab, a monoclonal antibody against receptor activator of nuclear factor kappa-B ligand (RANKL)) might have effects on subchondral bone, particularly in the phase of elevated bone remodelling and hypomineralisation. Indeed, for risedronate a reduction in the urinary excretion of crboxy-terminal telepeptides of type II collagen (CTX II), usually regarded as a marker of cartilage breakdown, was found, but not on symptoms and/or radiological progression. ${ }^{10}$ For alendronate and strontium ranelate, a reduction in osteophytes and disc-space narrowing of the spine was found in a post-hoc analysis of studies with patients with osteoporosis. ${ }^{11} 12$ Additionally, in a small study with zoledronic acid, a reduction in BMLs was found. ${ }^{13}$ With another antiosteoporotic drug, strontiumranelate, which is not a pure antiresorptive drug, a reduction in joint space width was demonstrated in a 3 -year trial versus placebo. ${ }^{14}$

There are remaining questions how a primary effect on subchondral bone might have subsequent effects on cartilage metabolism, and on anatomical structural abnormalities such as disc-space narrowing, osteophytes and BMLs. Nevertheless, all these data point in the direction that BPs might have an effect on OA (progression).

Since denosumab is also an antiresorptive drug, it is plausible that it also might have favourable effects in OA.

HOW STRONG ARE THE DATA, IS BIAS BY INDICATION FULLY EXCLUDED?

Neogi et al used the The Health Improvement Network (THIN) database, in which data are collected by general practitioners in UK. Only patients with incident knee-OA were enrolled, and 2006 patients who initiated BPs at least 1 year after diagnosis of knee-OA were compared with 2006 propensity-matched 'non-BP initiators'. After a mean follow-up of 3 years, the crude incidence of KR was 22.0 per 1000 person-years among the initiators, vs 29.1 among the non-initiators, with an HR of 0.76 (95\% CI 0.59 to 0.93$)$. Obviously, the key question is whether this is a direct effect of BPs on the osteoarthritic joint, or might confounding by indication play a role? For example, do BP users have a more healthy lifestyle, or higher vitamin D levels, or a higher socioeconomic status, or less frailty, and so on?

Without doubt, the authors tried to minimise the effect of confounding by indication, by performing a propensity score-matched cohort study, and by sensitivity analysis for those without data on BMI, smoking and/oralcohol, which all support the protective effect of BPs. Even the potentially lower mortality risk for patients with BP use was calculated. Nevertheless, although these all seem to be optimal and robust, it is not possible to completely rule out the risk of bias, because unknown bias cannot be detected and thus not calculated.

\section{ARE THESE DATA IN LINE WITH OTHER STUDIES?}

Remarkably, this year Fu et al ${ }^{15}$ published a more or less comparable, nationwide, study from Taiwan. They observed more than $12000 \mathrm{BP}$ users and 123000 non-users, all with knee-OA, and they found a $24 \%$ reduction in the percentage of patients for KR (HR $0.75,95 \%$ CI 0.69 to 0.83 ), which is remarkably in line with the THIN data. The study from Taiwan is larger, which is reflected in the smaller CI. But the authors found also a doseeffect relation: in those individuals who used BPs for more than 2 years and with a medication possession ratio $>80 \%$, the risk reduction for a KR was even larger: HR 0.66 (95\% CI 0.43 to 0.95 ). Moreover, the authors observed over a 5 -year follow-up period a significantly greater reduction in the use of non-steroidal anti-inflammatory drugs, acetaminophen and glucosamine in the BP users versus the non-users $(\mathrm{P}<0.001)$.

\section{WHAT ARE THE CONSEQUENCES?}

Obviously, the data from Neogi et al with the UK database and the data from Fu et al with the larger Taiwan database are in line 
with each other, which is very important because these data validate each other. Since both studies suggest that BPs reduce the percentage of patients with knee-OA who need a KR with around 25\%, this might indicate that patients with kneeOA who also have osteoporosis and an elevated fracture risk should preferably be treated with a BP. It is too early to conclude that BPs should also be prescribed in patients with knee-OA without osteoporosis, since prospective data on randomised studies in patients with knee-OA in which reduction in patients with KRs who are BP users versus placebo are lacking.

It is important to realise that both studies have the same case-control design, so it cannot completely be ruled out that they may have both the same structural limitation of confounding by indication in its design. Nevertheless, the larger reduction in KRs in patients with greater exposure to BPs is a strong argument in favour of a drug-induced effect.

What would be the next step? Given the paucity of therapeutic options in OA, these data strongly suggest that BPs have a favourable effect on the natural course of OA, which might have important consequences for our patients and for societies, since reduction or postponing of KRs might have large financial consequences. In my opinion, the data should preferably be confirmed in a randomised controlled trial in which elderly patients with incident knee-OA should be randomised to a BP (or another antiresorptive drug, such as denosumab) versus placebo. Because of the placebo arm, patients with high fracture risk resulting from generalised osteoporosis should be excluded, for ethical reasons. As a consequence, it could be that patients with osteoporotic subchondral bone will be excluded. However, a large sample size and long study duration will be necessary, unless we are able to select patients with OA with osteochondral bone that is vulnerable to the positive effects of BPs, thus with a high bone turnover and some hypomineralisation. Unfortunately, reliable, quantitative and validated measurements are even with new techniques as dual $\mathrm{x}$ ray absorptiometry (DXA), CT scan and/or MRI not yet possible.

To summarise, two comparable and high-quality studies have documented and suggested that BP use is associated with a $25 \%$ reduction in KRs. Since KR is a clinically relevant and hard endpoint, these studies hopefully might stimulate further research in the role of subchondral bone in OA, which is still urgently needed given the paucity of therapeutic options in OA.

\section{Competing interests None declared.}

Provenance and peer review Commissioned; externally peer reviewed.

(c) Article author(s) (or their employer(s) unless otherwise stated in the text of the article) 2018. All rights reserved. No commercial use is permitted unless otherwise expressly granted.

\section{A) Check for updates}

To cite Lems WF. Ann Rheum Dis

2018;77:1247-1248.

\section{CLinked}

- http://dx.doi.org/10.1136/annrheumdis-2017211811

Ann Rheum Dis 2018;77:1247-1248.

doi:10.1136/annrheumdis-2017-212364

\section{REFERENCES}

1 Allen KD, Golightly YM. Epidemiology of osteoarthritis: state of the evidence. Curr Opinion Rheumatology 2015;3:276-73.

2 Fernandes L, Hagen KB, Bijlsma JW, et al. EULAR recommendations for the non-pharmacological core management of hip and knee osteoarthritis. Ann Rheum Dis 2013;72:1125-35.

3 Reginster JY, Deroisy R, Rovati LC, et al. Long-term effects of glucosamine sulphate on osteoarthritis progression: a randomised, placebo-controlled clinical trial. Lancet 2001:357:251-6.

4 Kahan A, Uebelhart D, De Vathaire F, et al. Longterm effects of chondroitins 4 and 6 sulfate on knee osteoarthritis: the study on osteoarthritis progression prevention, a two-year, randomized, double-blind, placebo-controlled trial. Arthritis Rheum 2009;60:524-33.

5 Hügle T, Geurts J. What drives osteoarthritis? synovial versus subchondral bone pathology. Rheumatology 2017:56:1461-71.

6 Bijlsma JW, Berenbaum F, Lafeber FP. Osteoarthritis: an update with relevance for clinical practice. Lancet 2011;377:2115-26.

7 Roman-Blas JA, Castañeda S, Largo R, et al. An OA phenotype may obtain major benefit from bone-acting agents. Semin Arthritis Rheum 2014;43:421-8

8 Neogi T, Li S, Peloquin C, et al. Effect of bisphosphonates on knee replacement surgery. Ann Rheum Dis 2018;77:92-7.

9 Bultink IE, Lems WF. Osteoarthritis and osteoporosis: what is the overlap? Curr Rheumatol Rep 2013;15:328

10 Bingham CO III, Buckland Wright JC, Garnero P, et al. Effect of risedronate on joint structure and symptoms of knee osteoarthritis: results from the BRISK randomised controlled trial. Arthritis Rheum 2006:54:3494-407.

11 Neogi T, Nevitt MC, Ensrud KE, et al. The effect of alendronate on progression of spinal osteophytes and disc-space narrowing. Ann Rheum Dis 2008:67:1427-30.

12 Bruyere O, Delferriere D, Roux C, et al. Effects of strontium ranelate on spinal osteoarthritis progression. Ann Rheum Dis 2008;67:335-9.

13 Laslett LL, Doré DA, Quinn SJ, et al. Zoledronic acid reduces knee pain and bone marrow lesions over 1 year: a randomised controlled trial. Ann Rheum Dis 2012;71:1322-8

14 Reginster JY, Badurski J, Bellamy N, et al. Efficacy and safety of strontium ranelate in the treatment of knee osteoarthritis: results of a double-blind, randomised placebo-controlled trial. Ann Rheum Dis 2013;72:179-86

15 SH F, Wang CYm, Yang RS, et al. Bisphosphonate use and the risk of undergoing total knee artrhoplasty in osteoporotic patients with osteoarthritis. A Nationwide Cohort Study in Taiwan. J Bone Joint Surgery Am 2017;99:838-46. 